\title{
Accuracy of real-time PCR, Gram stain and culture for Streptococcus pneumoniae, Neisseria meningitidis and Haemophilus influenzae meningitis diagnosis
}

Henry M Wu ${ }^{1,5^{*}}$, Soraia M Cordeiro², Brian H Harcourt ${ }^{1}$, Maria da Gloria S Carvalho ${ }^{1}$, Jailton Azevedo², Tainara Q Oliveira ${ }^{3}$, Mariela C Leite ${ }^{3}$, Katia Salgado², Mitermayer G Reis², Brian D Plikaytis ${ }^{1}$, Thomas A Clark 1 , Leonard W Mayer ${ }^{1}$, Albert I Ko ${ }^{2,4}$, Stacey W Martin ${ }^{1}$ and Joice N Reis ${ }^{2,3}$

\begin{abstract}
Background: Although cerebrospinal fluid (CSF) culture is the diagnostic reference standard for bacterial meningitis, its sensitivity is limited, particularly when antibiotics were previously administered. CSF Gram staining and real-time PCR are theoretically less affected by antibiotics; however, it is difficult to evaluate these tests with an imperfect reference standard.

Methods and findings: CSF from patients with suspected meningitis from Salvador, Brazil were tested with culture, Gram stain, and real-time PCR using S. pneumoniae, N. meningitidis, and H. influenzae specific primers and probes. An antibiotic detection disk bioassay was used to test for the presence of antibiotic activity in CSF. The diagnostic accuracy of tests were evaluated using multiple methods, including direct evaluation of Gram stain and real-time PCR against CSF culture, evaluation of real-time PCR against a composite reference standard, and latent class analysis modeling to evaluate all three tests simultaneously.

Results: Among 451 CSF specimens, 80 (17.7\%) had culture isolation of one of the three pathogens (40 S. pneumoniae, $36 \mathrm{~N}$. meningitidis, and 4 H. influenzae), and 113 (25.1\%) were real-time PCR positive (51 S. pneumoniae, $57 \mathrm{~N}$. meningitidis, and $5 \mathrm{H}$. influenzae). Compared to culture, real-time PCR sensitivity and specificity were $95.0 \%$ and $90.0 \%$, respectively. In a latent class analysis model, the sensitivity and specificity estimates were: culture, $81.3 \%$ and 99.7\%; Gram stain, 98.2\% and 98.7\%; and real-time PCR, 95.7\% and 94.3\%, respectively. Gram stain and real-time PCR sensitivity did not change significantly when there was antibiotic activity in the CSF.
\end{abstract}

Conclusion: Real-time PCR and Gram stain were highly accurate in diagnosing meningitis caused by S. pneumoniae, N. meningitidis, and H. influenzae, though there were few cases of H. influenzae. Furthermore, real-time PCR and Gram staining were less affected by antibiotic presence and might be useful when antibiotics were previously administered. Gram staining, which is inexpensive and commonly available, should be encouraged in all clinical settings.

Keywords: Bacterial meningitis, Diagnostic test evaluation, Real-time PCR, Streptococcus pneumoniae Neisseria meningitidis, Haemophilus influenzae

\footnotetext{
* Correspondence: hmwu@emory.edu

'Division of Bacterial Diseases, National Center for Immunization and Respiratory Diseases, CDC, 1600 Clifton Road, Atlanta, GA 30333, USA

${ }^{5}$ Present address: 550 Peachtree Street NE, MOT 7, Atlanta, GA 30308, USA

Full list of author information is available at the end of the article
} 


\section{Background}

Despite advances in management, bacterial meningitis remains a severe infection with high rates of morbidity and mortality [1]. Early clinical suspicion and implementation of appropriate antimicrobial therapy are critical to minimize adverse outcomes. Cerebrospinal fluid (CSF) culture is considered the diagnostic reference standard for bacterial meningitis, and bacterial isolation is important for antimicrobial susceptibility testing and molecular epidemiology. However, CSF culture requires at least a day or more, and has limited sensitivity. Reported CSF culture sensitivities typically range between $70 \%$ to $90 \%$ [2-4], with variation in case inclusion criteria, patient characteristics, laboratory practices, and spectrum of bacterial pathogens likely contributing to the observed differences. Administration of antibiotics prior to lumbar puncture is a common situation leading to decreased culture yield [1,2,5-7]. In practice, lumbar puncture can be delayed, resulting in antibiotics given prior to CSF collection. Administration of oral antibiotics prior to admission might also occur if the patient previously sought outpatient treatment or if antibiotics are available without a physician prescription.

Gram staining, a mainstay of bacterial meningitis diagnosis, is widely available, inexpensive and rapid [1]. Reported sensitivities of CSF Gram staining range from $60 \%$ to $90 \%$ $[1,6-9]$, although it can difficult to assess its actual performance with an imperfect reference standard. Real-time polymerase chain reaction (RT-PCR) of CSF has been suggested as a rapid diagnostic test for bacterial meningitis [1,10-12], and amplification of DNA from non-viable bacteria could potentially facilitate diagnosis in culture negative cases [12].

We evaluated the clinical accuracy of RT-PCR and Gram staining for the diagnosis of Streptococcus pneumoniae, Neisseria meningitidis, and Haemophilus influenzae meningitis in patients in Salvador, Brazil. An antibiotic detection disk bioassay was also performed on CSF to detect the presence of antibiotic activity. Since the imperfect sensitivity of CSF culture presents a challenge when using it to evaluate tests that might be more sensitive, sensitivity and specificity estimates from multiple methods were compared. RT-PCR and Gram stain were compared to a culture reference standard, and RT-PCR was further evaluated using a composite reference standard (CRS) of culture and Gram stain. A CRS addresses an imperfect reference standard by adding a second test [13]. Latent class analysis (LCA) models were used to evaluate all three tests. LCA modeling can assess multiple diagnostic tests simultaneously and has been used to evaluate tests for other infections with imperfect reference standards [13,14].

\section{Methods}

\section{Ethics statement}

This protocol was approved by the institutional review boards of FIOCRUZ and Weill Medical College of Cornell
University, and the protocol was exempt from requiring institutional review board approval at the US Centers for Disease Control and Prevention (CDC) because it was determined to be non-human research for CDC collaborators. Written informed consent was obtained from all participants of this study, except in the situation where the participant was unable to give written informed consent due to illness. When the participant was unable give consent due to illness, written informed consent was obtained from the subject's legally authorized representative.

\section{Study population and routine clinical testing}

According to state health department guidelines, suspected cases of meningitis in the metropolitan region of Salvador, a city of over 2 million inhabitants in northeast Brazil, are evaluated at the emergency department of a single public infectious diseases hospital (Hospital Couto Maia, Salvador, Bahia) [15]. As per routine hospital clinical guidelines, lumbar puncture was performed on all patients with suspected acute meningitis, defined as fever $>38^{\circ} \mathrm{C}$ of duration $<21$ days and physical exam findings of either meningismus or altered mental status. CSF specimens were tested according to laboratory guidelines to the extent possible with available specimen amount. Routine testing of CSF included leukocyte, glucose, and protein counts. Gram staining was indicated for all CSF specimens with elevated leukocyte counts $\left(>5 \times 10^{6}\right.$ cells $/ \mathrm{L}$, or $>15 \times 10^{6}$ cells $/ \mathrm{L}$ for infants aged < 30 days old), polymorphonuclear cell (PMN) predominance ( $>50 \%$ of leukocytes), or abnormal chemistry results (glucose $<2.2 \mathrm{mmol} / \mathrm{L}$, or protein $>0.65 \mathrm{~g} / \mathrm{L}$ ). Culture was indicated for CSF specimens with a leukocyte count $>1000 \times 10^{6}$ cells $/$ L, PMN predominance, or abnormal chemistry results as defined above. Gram staining and cultures were also performed outside of routine guidelines when requested by the clinician or clinical microbiologist. Latex agglutination testing for bacterial antigens was variably performed on CSF specimens depending on test kit availability (various commercial kits used) and clinician request.

As a part of an active, hospital-based surveillance program for acute meningitis established in Salvador [15], surveillance staff reviewed daily laboratory records to identify patients meeting one of the following surveillance criteria: total CSF leukocyte count $>100 \times 10^{6}$ cells $/ \mathrm{L}$ or abnormal CSF chemistry as defined above. A standardized case report form was used to collect clinical and epidemiologic data from medical records and when possible, patient or family interview. If the volume of CSF collected was more than adequate for routine testing, $0.5-1 \mathrm{~mL}$ was saved for testing with RT-PCR and the antimicrobial detection assay at the Oswaldo Cruz Foundation (FIOCRUZ, Salvador, Brazil). 
Patients meeting surveillance criteria from April 10, 2006 through December 31, 2008 were considered for this analysis. Patients with specimens without results for any of the three tests under evaluation (i.e., missing results or not tested with CSF Gram stain, culture or RT-PCR for any reason) were excluded. Patients not admitted to the emergency department or inpatient ward of Couto Maia Hospital were also excluded (i.e., CSF specimens obtained from patients evaluated elsewhere but submitted to the Couto Maia Hospital laboratory).

\section{Real-time PCR}

DNA was extracted from CSF with the QIAGEN DNA Mini kit (QIAGEN Inc., Valencia, CA). To facilitate full lysis of gram-positive bacteria, a modified protocol was used. Briefly, $200 \mu \mathrm{l}$ of CSF was added to $100 \mu \mathrm{l}$ of Tris-EDTA buffer containing $0.04 \mathrm{~g} / \mathrm{ml}$ lysozyme and $75 \mathrm{U} / \mathrm{ml}$ of mutanolysin (Sigma, St. Louis, MO), and the mixture was incubated for 1 hour in a $37^{\circ} \mathrm{C}$ water bath. All subsequent steps were performed according to the QIAGEN DNA Mini kit manufacturer protocol [16]. DNA was eluted in $100 \mu \mathrm{l}$ of QIAGEN elution buffer and stored at $-20^{\circ} \mathrm{C}$.

The lytA gene of $S$. pneumoniae [16], ctrA gene of $N$. meningitidis [17], and bexA gene of $H$. influenzae [18] were used as species-specific targets, and a positive result was indicated by amplification with an exponential increase in fluorescence in separate reactions. The primer and probe sequences are listed in Table 1 . The assays were carried out in a $25 \mu \mathrm{l}$ reaction volume with $2 \mu \mathrm{l}$ of sample DNA and were performed by use of the TaqMan Universal Master Mix kit (Applied Biosystems, Foster City, CA), according to manufacturer instructions. No-template controls, S. pneumoniae, N. meningitidis, and $H$. influenzae-positive controls, and extracted water negative controls were included in every run. DNA was amplified with the 7500 Real Time PCR system (Applied Biosystems) by using the following temperature program: $50^{\circ} \mathrm{C}$ for $2 \mathrm{~min}, 95^{\circ} \mathrm{C}$ for $10 \mathrm{~min}$, followed by 50 cycles of $95^{\circ} \mathrm{C}$ for $15 \mathrm{~s}$ and $60^{\circ} \mathrm{C}$ for $1 \mathrm{~min}$. Amplification data were analyzed by instrument software (Applied Biosystems). Positive specimens were defined as those with a cycle threshold $(\mathrm{CT})$ value of $<36$. Negative specimens were defined as those with $\mathrm{CT}$ values $>40$. Specimens with $\mathrm{CT}$ values from 36-40 were deemed equivocal and rerun at 1:4 dilution to dilute any PCR inhibitors that might be present. The rerun CT value was interpreted with the same cutoffs; specimens with repeat $\mathrm{CT}$ values still in the equivocal range were considered indeterminate. If a specimen was positive for more than one gene target, RT-PCR was repeated to confirm the results. If only one target was positive on repeat testing, the specimen was considered positive for that target only. Staff conducting the RT-PCR assays were blinded to the results of routine laboratory testing and other patient clinical data.

\section{Antibiotic detection}

An antibiotic detection bioassay was performed to detect antibiotic presence in CSF specimens. Briefly, an inoculum of a pure culture of a pan-sensitive Micrococcus luteus strain (ATCC 7468) was calibrated to a McFarland standard of 0.5. A cotton-tipped sterile applicator was used to inoculate a nutrient agar plate for confluent growth. The inoculum was allowed to dry for 5 minutes before placing two sterile 6-mm filter paper disks on the plate approximately $3 \mathrm{~cm}$ apart. $20 \mu \mathrm{l}$ of CSF was placed on one disk and $20 \mu \mathrm{l}$ of sterile saline was placed on the other. Plates were incubated at $37^{\circ} \mathrm{C}$ in air atmosphere for 18-24 hours and examined for a zone of inhibition. A positive result was defined as the presence of any inhibition zone around the CSF-inoculated disk.

\section{Data analyses}

For comparison of patients tested with RT-PCR with those that were not tested, categorical variables were compared using the chi-square or Fisher's exact test. Continuous variables were compared using the Wilcoxon-Mann-Whitney test. Tests of significance were 2-tailed and a $p \leq 0.05$ was considered to be significant.

Table 1 Real-time PCR primers and probes [16-18]

\begin{tabular}{llc}
\hline Oligonuclotide & Sequence & Final conc. (nM) \\
\hline ctrA forward & 5'-TGTGTCCGCTATACGCCATT-3' & 300 \\
ctrA reverse & 5'-GCCATATTCACACGATATACC-3' & 900 \\
ctrA probe & 5'-FAM-AACCTTGAGCAA"T"CCATTTATCCTGACGTTCT-3'-SpC6* & 100 \\
bexA forward & 5'-TGCGGTAGTGTTAGAAAATGGTATTATG-3' & 600 \\
bexA reverse & 5'-GGACAAACATCACAAGCGGTA-3' & 600 \\
bexA probe & 5'-FAM-ACAAAGCGTATCAA"T"ACTACAACGAGACGCAAAAA-3'-SpC6* & 100 \\
lytA forward & 5'-ACGCAATCTAGCAGATGAAGCA-3' & 200 \\
lytA reverse & 5'-TCGTGCGTTTAATTCCAGCT & 200 \\
lytA probe & 5'-FAM-TGCCGAAAACGCTTGATACAGGGAG-3'-BHQ1 & 200 \\
\hline
\end{tabular}

* The quencher BHQ-1 is attached to the internal thymine residue " $\mathrm{T}$ ". 
For sensitivity and specificity analyses, a positive test was defined as a test result indicative of S. pneumoniae, $N$. meningitidis, or H. influenzae in CSF. For culture, a positive result was defined as the isolation of one of the three pathogens. A positive Gram stain result was defined as the report of gram-positive diplococci (suggestive of $S$. pneumoniae), gram-negative diplococci (suggestive of $N$. meningitidis), or gram-negative pleomorphic coccobacilli (suggestive of $H$. influenzae). All other culture and Gram stain results, including the absence of bacteria or the presence of other bacterial species (in the absence of S. pneumoniae, N. meningitidis, and $H$. influenzae), were considered a negative test result. Positive RT-PCR assays were defined as those positive for one of the three pathogens as described above. Specimens with indeterminate RT-PCR results were considered negative in all analyses. Species-specific estimates of test accuracy were also performed with positive test results defined as those indicative of the specific species being considered, and a negative result was defined as the absence of any result indicative of that particular species (i.e., no bacteria present or presence of other bacterial species in the absence of the species being considered).

Sensitivity and specificity of RT-PCR and Gram staining were calculated using classical $2 \times 2$ validation analysis using a CSF culture reference standard. RT-PCR performance was also evaluated using $2 \times 2$ validation with a CRS of CSF culture and Gram stain. For each patient, a positive CRS required the presence of a positive culture or Gram stain result. A negative CRS required negative culture and Gram stain results as defined above. A 95\% exact binomial confidence interval was calculated for each parameter derived from classical validation and CRS analyses.

Latent class analyses (LCA) was used to estimate the clinical sensitivity and specificity of culture, Gram stain, and RT-PCR simultaneously. LCA fits a model to data from multiple diagnostic tests to model the prevalence of a latent class (disease status) in the analyzed population. Sensitivity and specificity estimates are based on conditional response probabilities predicted by the model [14]. For the primary LCA model, the latent class was the presence or absence of meningitis caused by $S$. pneumoniae, N. meningitidis, or $H$. influenzae. For each model, a bootstrap $p$ value greater than 0.1 was considered indicative of a good fit with the observed data. Correlation between diagnostic tests not explained by the model were considered absent if the bivariate residual between test pairs was $<3.84$. Standard errors (SE) for each test parameter and disease prevalence estimate were provided by the LCA model and were used to calculate $95 \%$ confidence intervals (estimate +/$1.96 * \mathrm{SE})$. LCA was performed using Latent Gold 4.0 (Statistical Innovations; Belmont, MA) and all other analyses were performed using SAS 9.2 (SAS Institute Inc.; Cary, NC).

\section{Results}

\section{Study population}

From April 10, 2006 through December 31, 2008, a total of 2,554 patients met surveillance criteria, and 1,661 of these patients were evaluated at Couto Maia Hospital and had complete CSF Gram stain and culture results. Among these study eligible patients, RT-PCR was done on 451 (27.2\%) of their specimens (Figure 1). Comparison of patients with CSF tested with RT-PCR with those not tested with RT-PCR showed that patients whose CSF was tested with RT-PCR were more likely to be transferred from another hospital, have an altered mental status, have higher CSF leukocyte counts, have lower CSF glucose levels, have bacteria on Gram stain, and have an isolate from CSF culture (Table 2).

\section{Test results}

Among 451 patients with CSF Gram stain, culture, and RT-PCR done, 80 (17.7\%) had CSF culture isolation of $S$. pneumoniae, $N$. meningitidis, or $H$. influenzae (Tables 2 and 3). One hundred patients (22.2\%) had a CSF Gram stain suggestive of $S$. pneumoniae, N. meningitidis, or H. influenzae. RT-PCR of CSF was positive for 113 (25.1\%) patients, of which 37 (32.7\%) had negative culture results (as per study definition), 39 (34.5\%) had S. pneumoniae isolated, 34 (30.0\%) had N. meningitidis isolated, and 3 (2.7\%) had H. influenzae isolated (Figure 1). Four specimens had indeterminate RT-PCR results (two for $\operatorname{lyt} \mathrm{A}$ and two for $\operatorname{ctr} A$ ), and these results were considered negative in all analyses. RT-PCR was negative in all 20 CSF specimens that with culture isolation of bacteria other than S. pneumoniae, $N$. meningitidis, or H. influenzae. Among 96 specimens with a positive result for two or more of the diagnostic tests being considered, only one had positive results suggestive of different species when interpreted with study definitions. This specimen had a sterile culture, positive RT-PCR for $N$. meningitidis, and a Gram stain read as having gram-positive diplococci.

\section{Sensitivity and specificity estimates}

Sensitivity and specificity estimates are summarized in Table 4. Using a culture reference standard, sensitivity and specificity of RT-PCR were $95.0 \%$ (95\% CI 87.7-98.6\%) and 90.0\% (95\% CI 86.5-92.9\%), and Gram stain sensitivity and specificity were $97.5 \%$ (95\% CI 91.3-99.7\%) and 94.1\% (95\% CI 91.2-96.3\%), respectively. RT-PCR sensitivity decreased and specificity increased to $94.3 \%$ (95\% CI 91.3-96.5\%) when compared against the CRS. In a LCA model with CSF culture, Gram stain, and RT-PCR (Table 4), the modeled prevalence of meningitis caused by S. pneumoniae, $N$. meningitidis, or $H$. influenzae was $21.5 \%$ (95\% CI 17.7- 


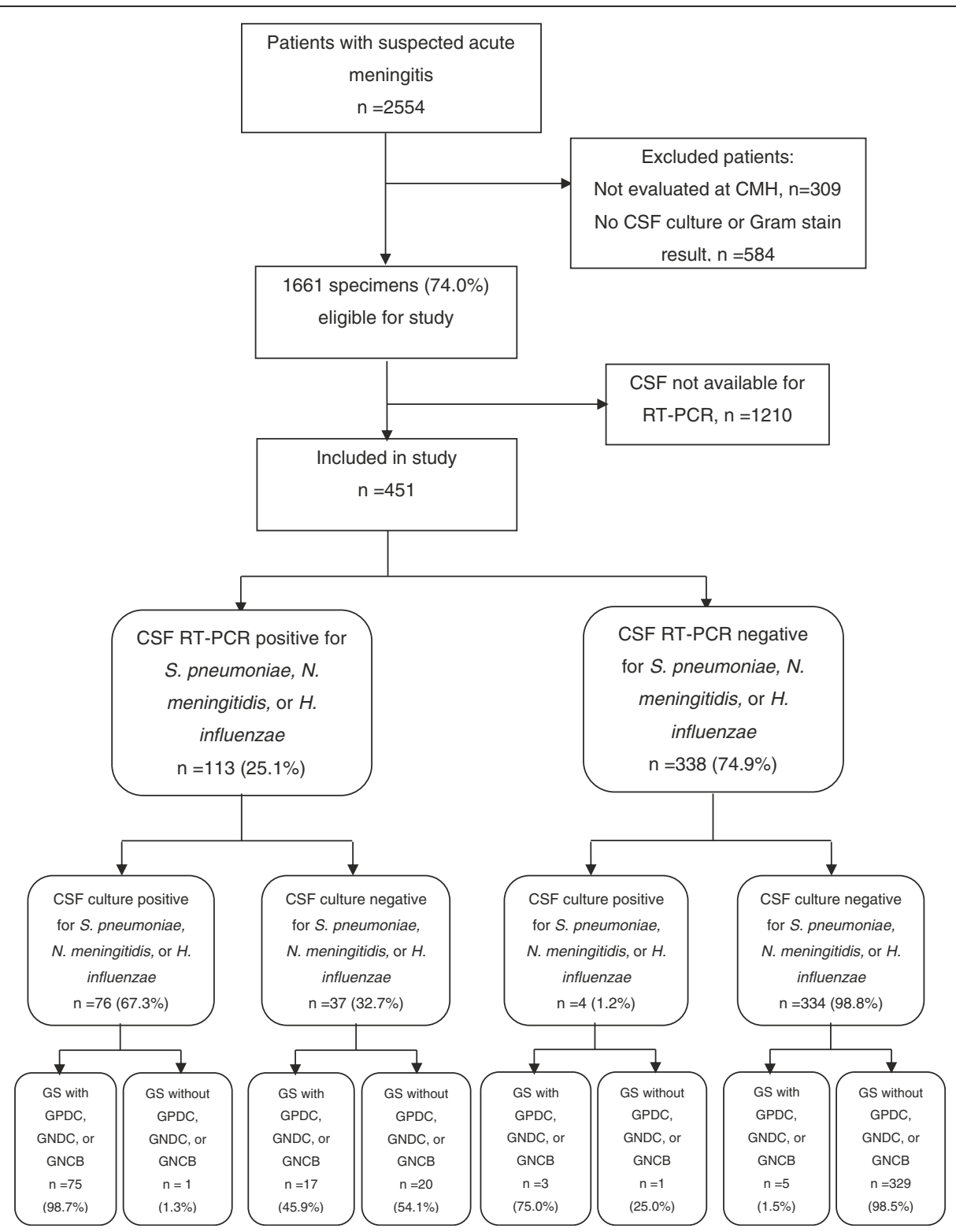

Figure 1 Participant inclusion, RT-PCR, and culture results flow diagram. Following exclusion of patients not meeting inclusion criteria and specimens that were unavailable for RT-PCR testing, the RT-PCR and culture results were analyzed for 451 specimens. Abbreviations: CMH, Couto Maia Hospital; CSF, cerebrospinal fluid; RT-PCR, real-time polymerase chain reaction; GS, Gram stain; GPDC, gram-positive diplococci; GNDC, gram-negative diplococci; GNCB, gram-negative pleomorphic coccobacilli.

25.4), and culture sensitivity (81.3\%; 95CI 73.2-89.3) was less than that of Gram stain (98.2\%; 95\% CI 95.2$100.0 \%)$ and RT-PCR (95.7\%; 95\% CI 91.2-100.0\%). RTPCR specificity in the LCA model was $94.3 \%$ (95\% CI 91.9-96.8\%).

Among 439 specimens tested with the antimicrobial detection disk bioassay, 98 (22.3\%) tested positive. Sensitivity and specificity estimates following stratification of patients based on the bioassay result are shown in Table 4. There was minimal decrease in Gram stain and RT-PCR sensitivity in specimens with evidence of antimicrobial activity, while there was a large decrease in RT-PCR specificity among specimens with evidence of antimicrobial activity. Culture sensitivity decreased among specimens with evidence of antimicrobial activity. Species-specific test parameters for S. pneumoniae and $N$. meningitidis were similar to those determined for all three pathogens considered together (Table 4). $H$. influenzae-specific test parameters were not calculated due to the low numbers of positive tests.

All LCA models achieved adequate fit based on bootstrap $p$ values criteria, and correlations between diagnostic 
Table 2 Demographic, clinical, and laboratory characteristics of patients included in this study

\begin{tabular}{lccc}
\hline $\begin{array}{l}\text { Demographic and clinical } \\
\text { characteristics }\end{array}$ & $\begin{array}{c}\text { Analyzed } \\
\text { cases } \\
(\mathbf{n}=\mathbf{4 5 1})\end{array}$ & $\begin{array}{c}\text { Cases without } \\
\text { RT-PCR } \\
(\mathbf{n}=1210)\end{array}$ \\
\hline Male (\%) & $284(63.0)$ & $731(60.8)$ & \\
$\begin{array}{l}\text { Median age in years (range) } \\
11(0-78)\end{array}$ & $11(0-83)$ & \\
$\begin{array}{l}\text { Days with symptoms before } \\
\text { admission (median, range) }\end{array}$ & $2(0-60)$ & $2(0-120)$ & \\
$\begin{array}{l}\text { Admitted to intensive care } \\
\text { unit (\%) }\end{array}$ & $56(13.4)$ & $104(10.3)$ & \\
$\begin{array}{l}\text { Altered mental status on } \\
\text { admission (\%) }\end{array}$ & $133(33.1)$ & $262(25.9)$ & $p=0.007$ \\
$\begin{array}{l}\text { Died (\%) } \\
\text { Transferred from another }\end{array}$ & $270(60.8)$ & $634(52.6)$ & $p=0.003$
\end{tabular}

hospital (\%)

\section{CSF cell counts and chemistry}

Leukocyte count (\%)

$<10 \times 10^{6}$ cells/L

$10-99 \times 10^{6}$ cells/L

$100-999 \times 10^{6}$ cells/L

$1000-9,999 \times 10^{6}$ cells/L

$107(23.8)$

$\geq 10,000 \times 10^{6}$ cells $/ \mathrm{L}$

77 (17.1)

$302(67.1)$

$242(53.7)$

$136(30.2)$

$>1.00 \mathrm{~g} / \mathrm{L}$ protein $(\%)$

$\leq 1.9 \mathrm{mmol} / \mathrm{L}$ glucose (\%)

\section{Gram stain results}

Gram stain with any bacteria (\%)

GPDC, GNDC, or GNCB (\%)

GPDC (\%)

GNDC (\%)

GNCB (\%)

Gram stain with bacteria of other morphology

\section{Culture results}

Positive for any bacteria (\%)

S. pneumoniae, $N$.

meningitidis, or $\mathrm{H}$. influenzae (\%)

$\begin{array}{lcc}\text { S. pneumoniae (\%) } & 40(8.9) & 61(5.0) \\ \text { N. meningitidis (\%) } & 36(8.0) & 67(5.5) \\ \text { H. influenzae (\%) } & 4(0.9) & 15(1.2) \\ \begin{array}{l}\text { Streptococcus agalactiae (\%) } \\ \text { Other Streptococcus species }\end{array} & 1(0.4) & 2(0.2) \\ \begin{array}{l}(\%) \\ \text { Staphylococcus species (\%) }\end{array} & 5(1.1) & 4(0.3) \\ \begin{array}{l}\text { Escherichia coli (\%) } \\ \text { Listeria monocytogenes (\%) }\end{array} & 2(0.4) & 5(0.4) \\ \end{array}$

$p=0.001$

$7(0.6)$

$17(1.5)$

$743(64.3)$

$262(22.7)$

$127(11.0)$

$725(62.7)$

$621(51.5)$

$303(25.1)$

$p=0.04$

$116(25.7) \quad 208(17.2) \quad p<0.0001$

$100(22.2) \quad 172(14.2)$

$49(10.9) \quad 80(6.6)$

$46(10.2) \quad 80(6.6)$

$5(1.1) \quad 12(1.0)$

$16(3.6) \quad 36(3.0)$

$100(22.2) \quad 178(14.7) \quad p=0.0003$

$80(17.7) \quad 143(11.8)$
Table 2 Demographic, clinical, and laboratory characteristics of patients included in this study (Continued)

\begin{tabular}{|c|c|c|}
\hline Salmonella species (\%) & $1(0.2)$ & 0 \\
\hline Other bacteria & $8(1.8)$ & $20(1.7)$ \\
\hline \multicolumn{3}{|l|}{ RT-PCR result } \\
\hline Any positive (\%) & $113(25.1)$ & - \\
\hline S. pneumoniae (\%) & $51(11.3)$ & - \\
\hline N. meningitidis (\%) & 57 (12.6) & - \\
\hline H. influenzae (\%) & $5(1.1)$ & - \\
\hline \multicolumn{3}{|c|}{$\begin{array}{l}\text { Patients included in this study are compared to those who were excluded due } \\
\text { to the absence of CSF RT-PCR testing. } \\
\text { Note. Denominators may vary due to incomplete data. } \\
\text { Abbreviations: RT-PCR, real-time polymerase chain reaction; CSF, cerebrospinal } \\
\text { fluid; PMN, polymorphonuclear leukocyte; GPDC, gram-positive diplococci; } \\
\text { GNDC, gram-negative diplococcic; GNCB, gram-negative pleomorphic } \\
\text { coccobacilli. }\end{array}$} \\
\hline
\end{tabular}

tests were adequately explained by the models based on bivariate residual criteria.

\section{Patients with isolated RT-PCR-positive CSF}

Patients with CSF specimens that were positive by RT-PCR with negative cultures and Gram stains for S. pneumoniae, $N$. meningitidis, and $H$. influenzae $(\mathrm{n}=20)$ are compared with patients with all negative and all positive test results in Table 5. Test results for the patients with an isolated positive RT-PCR result were most similar with those from patients with positive results for all three diagnostic tests. Latex agglutination test results for bacterial antigens were available for 13 of the patients with an isolated positive RT-PCR result. Five of these latex agglutination tests were positive, and all positive results were concordant with the pathogen identified by RT-PCR.

\section{Discussion}

RT-PCR and Gram staining were highly accurate for S. pneumoniae, $N$. meningitidis, and $H$. influenzae meningitis diagnosis with sensitivity and specificity estimates above $90 \%$ in all analytic approaches. Although CSF culture is considered the diagnostic reference standard for bacterial meningitis, its limited sensitivity often results in an inability to confirm the diagnosis and target antimicrobial therapy. The imperfect sensitivity of culture also presents a difficulty when evaluating other tests that are potentially more sensitive. Composite reference standards and LCA modeling, which can estimate test performance parameters of multiple tests simultaneously, can be helpful in addressing this problem. We are unaware of previous published studies what have used LCA modeling to evaluate the clinical accuracy of tests for acute bacterial meningitis, an approach that requires no assumptions about the accuracy of CSF culture and furthermore, allows us to 
Table 3 Test result patterns for CSF culture, Gram stain, and RT-PCR for analyzed patients (total $n=451$ )

\begin{tabular}{lccc}
\hline Culture & Gram stain & RT-PCR & $\mathbf{n}$ \\
\hline- & - & - & 329 \\
- & - & + & 20 \\
- & + & - & 5 \\
- & + & + & 17 \\
+ & - & - & 1 \\
+ & - & + & 1 \\
+ & + & - & 3 \\
+ & + & + & 75 \\
\hline
\end{tabular}

Note. Positive test results (+) for culture or RT-PCR defined as identification of N. meningitidis, S. pneumoniae, or $H$. influenzae. Positive results (+) for Gram stains defined as report of bacteria with characteristic morphology and staining suggestive of $N$. meningitidis, S. pneumoniae, or $H$. influenzae. Any result not meeting the positive result definitions are denoted as negative (-).

also compare the sensitivity of CSF culture with the comparison tests. The sensitivities of RT-PCR and Gram staining were over $95 \%$ and far exceeded the $81 \%$ sensitivity of culture when estimated using LCA models. LCA models stratified by results of the antibiotic detection bioassay also provide evidence that RT-PCR and Gram stain sensitivity are less affected by the presence of antibiotic activity in the CSF. This is consistent with a recent study that found the presence of antibiotic activity in CSF is a strong risk factor for a RT-PCR positive, culture-negative case [12]. These findings suggest that RT-PCR might be particularly useful in settings where patients often receive antibiotics prior to lumbar puncture.

Nucleic acid amplification tests such as PCR do not require viable bacteria for a positive assay and are generally considered to be highly sensitive [14]. Nevertheless, the presence of PCR inhibitors in clinical specimens can compromise sensitivity [19], and the presence of inhibitors might explain the imperfect RT-PCR sensitivity in this and other studies [1]. When compared to a culture reference standard, RT-PCR specificity in this study was $90 \%$. While contamination of RT-PCR reactions leading to falsepositive results is always a concern, stratification of subjects by the presence or absence of antibiotic activity in the CSF resulted in RT-PCR specificities of $56 \%$ and $96 \%$, respectively. This suggests that the observed RT-PCR specificity is negatively biased by the imperfect sensitivity of culture, particularly when antibiotics have been administered. RT-PCR specificities calculated using a CRS or LCA

Table 4 Sensitivity and specificity estimates for diagnosis of S. pneumoniae, N. meningitidis, or H. influenzae meningitis

\begin{tabular}{|c|c|c|c|c|c|c|c|}
\hline & & \multicolumn{2}{|c|}{ Culture Standard } & \multicolumn{2}{|c|}{$\mathrm{CRS}^{\mathrm{a}}$} & \multicolumn{2}{|c|}{ LCA Model $^{\mathrm{b}}$} \\
\hline & & Sens $(\%, 95 \mathrm{Cl})$ & Spec $(\%, 95 \mathrm{Cl})$ & Sens $(\%, 95 \mathrm{Cl})$ & Spec $(\%, 95 \mathrm{Cl})$ & Sens $(\%, 95 \mathrm{Cl})$ & Spec $(\%, 95 \mathrm{Cl})$ \\
\hline \multicolumn{8}{|c|}{ S. pneumoniae, $N$. meningitidis, or $H$. influenzae } \\
\hline \multirow[t]{3}{*}{ All patients, $n=451$} & Culture & na & na & na & na & $81.3(73.2-89.3)$ & $99.7(99.1-100.0)$ \\
\hline & Gram stain & $97.5(91.3-99.7)$ & $94.1(91.2-96.3)$ & na & na & $98.2(95.2-100.0)$ & $98.7(97.3-100.0)$ \\
\hline & RT-PCR & $95.0(87.7-98.6)$ & $90.0(86.5-92.9)$ & $91.2(83.9-95.9)$ & $94.3(91.3-96.5)$ & $95.7(91.2-100.0)$ & $94.3(91.9-96.8)$ \\
\hline \multirow[t]{3}{*}{ Antibiotic negative, $\mathrm{n}=341$} & Culture & na & na & na & na & $85.7(74.4-96.9)$ & $100.0(99.8-100.0)$ \\
\hline & Gram stain & $100.0(89.7-100.0)$ & $97.4(94.9-98.9)$ & na & na & $98.7(94.9-100.0)$ & $99.0(97.9-100.0)$ \\
\hline & RT-PCR & $97.1(84.7-99.9)$ & $96.1(93.3-98.0)$ & $90.5(77.4-97.3)$ & $97.7(95.2-99.1)$ & $95.8(89.1-100.0)$ & $97.7(95.9-99.4)$ \\
\hline \multirow[t]{3}{*}{ Antibiotic positive, $\mathrm{n}=98$} & Culture & na & na & na & na & $78.2(66.4-89.9)$ & $97.1(91.9-100.0)$ \\
\hline & Gram stain & $97.7(88.0-99.9)$ & $74.1(60.4-85.0)$ & na & na & $99.5(97.3-100.0)$ & $94.0(84.1-100.0)$ \\
\hline & RT-PCR & $93.2(81.3-98.6)$ & $55.6(41.4-69.1)$ & $91.4(81.0-97.1)$ & $70.0(53.5-83.4)$ & $95.3(89.0-100.0)$ & $70.2(56.2-84.2)$ \\
\hline \multicolumn{8}{|l|}{ S. pneumoniae only } \\
\hline \multirow[t]{3}{*}{ All patients, $n=451$} & Culture & na & na & na & na & $85.3(74.8-95.9)$ & $100.0(99.9-100.0)$ \\
\hline & Gram stain & $97.5(86.8-99.9)$ & $97.6(95.6-98.8)$ & na & na & $96.4(90.6-100.0)$ & $99.0(98.1-100.0)$ \\
\hline & RT-PCR & $97.5(86.8-99.9)$ & $97.1(95.0-98.5)$ & $90.0(78.2-96.7)$ & $98.5(96.8-99.5)$ & $96.4(90.6-100.0)$ & $98.6(97.4-99.7)$ \\
\hline \multicolumn{8}{|l|}{ N. meningitidis only } \\
\hline \multirow[t]{3}{*}{ All patients, $n=451$} & Culture & na & na & na & na & $77.9(65.4-90.4)$ & $99.8(99.3-100.0)$ \\
\hline & Gram stain & $97.2(85.5-99.9)$ & $97.4(95.3-98.7)$ & na & na & $98.7(94.8-100.0)$ & $99.6(98.9-100.0)$ \\
\hline & RT-PCR & 94.4 (81.3-99.3) & $94.5(91.8-96.5)$ & 91.5 (79.6-97.6) & $96.5(94.3-98.1)$ & $95.9(89.3-100.0)$ & 96.6 (94.8-98.3) \\
\hline
\end{tabular}

Performance parameters for the various tests are estimated using a CSF culture reference standard, composite reference standard (CRS), and latent class analysis (LCA) modeling with stratification by antimicrobial detection disk bioassay results.

Abbreviations: CRS, composite reference standard; LCA, latent class analysis; na, not applicable; $95 \% \mathrm{Cl}$, 95\% exact binomial confidence interval for culture reference standard and CRS parameters, or parameter estimate $+/-1.96 *$ standard error for LCA models.

${ }^{a}$ Composite reference standard defined as positive if CSF culture or Gram stain positive for S. pneumoniae, N. meningitidis, or $\mathrm{H}$. influenzae.

${ }^{b}$ For LCA model of all patients, disease prevalence was $21.5 \%(95 \% \mathrm{Cl} 17.7-25.4)$. For the model of antibiotic assay-negative patients, disease prevalence was $11.6 \%(95 \mathrm{Cl} 8.2-15.0)$. For the model of antibiotic assay-positive patients, disease prevalence was $55.8 \%(96 \mathrm{Cl} 45.4-66.1)$. For the model of $S$. pneumoniae diagnosis, disease prevelance was $10.4 \%$ (95Cl 7.5-13.2). For the model of $N$. meningitidis diagnosis, disease prevalence was $10.0 \%$ (95Cl 7.2-12.7). Bootstrap $p$ for all models $>0.1$. 
Table 5 Characteristics of patients with isolated positive RT-PCR results

\begin{tabular}{|c|c|c|c|}
\hline & \multicolumn{3}{|c|}{ Culture-Gram-PCR result $^{\mathrm{b}}$} \\
\hline & $\begin{array}{l}\text { All tests negative } \\
\qquad(n=329)\end{array}$ & $\begin{array}{l}\text { Isolated RT-PCR positive } \\
\qquad(\mathrm{n}=20)\end{array}$ & $\begin{array}{l}\text { All tests positive } \\
\qquad(n=75)\end{array}$ \\
\hline Age in years (median, range) & $9(0-64)$ & $11(0-63)$ & $15(0-78)$ \\
\hline Altered mental status (\%) & $66(22.6)$ & $6(40.0)$ & $47(66.2)$ \\
\hline Deaths (\%) & $27(9.1)$ & $2(10.5)$ & $10(14.3)$ \\
\hline Median WBC ( $\times 10^{6}$ cells $/ L$, range $)^{c}$ & $430(100-10,000)$ & $10,000(32-10,000)$ & $8,600(0-10,000)$ \\
\hline$>50 \%$ PMN cells (\%) & $186(56.7)$ & $18(90.0)$ & $72(96.0)$ \\
\hline Median protein $(\mathrm{g} / \mathrm{L} \text {, range })^{d}$ & $0.50(0.20-5.00)$ & $2.90(0.32-5.00)$ & $3.50(0.28-5.00)$ \\
\hline Median glucose (mmol/L, range) & $2.9(1.1-3.9)$ & $1.6(0.6-3.1)$ & $1.1(1.1-3.2)$ \\
\hline LAT positive (\% of total LAT performed) & $1(2.0)$ & $5(38.5)$ & 25 (80.6) \\
\hline Positive LAT concordant with RT-PCR (\%) & $\mathrm{n} / \mathrm{a}$ & $5(100.0)$ & $25(100.0)$ \\
\hline Antibiotic bioassay positive (\%) & $28(8.8)$ & $12(63.2)$ & $41(55.4)$ \\
\hline
\end{tabular}

Patients with isolated positive RT-PCR results are compared with patients with all negative or all positive culture, Gram stain, and RT-PCR test results. ${ }^{a}$

Abbreviations: WBC, white blood cells; PMN, polymorphonuclear; LAT, latex agglutination test; n/a, not applicable.

apatients with missing data exclude from calculations.

${ }^{b}$ Positive or negative for N. meningitidis, S. pneumoniae, or H. influenzae as defined in Methods.

${ }^{c}$ Maximum value truncated at $10,000 \times 10^{6}$ cells/L.

${ }^{\mathrm{d}}$ Maximum value truncated at $5.00 \mathrm{~g} / \mathrm{L}$.

${ }^{\mathrm{e}}$ Number and percentage of positive latex agglutination test results that were consistent with RT-PCR result.

modeling were higher, suggesting that these approaches can help address the bias introduced by the insensitivity of culture. Nonetheless, it appears that RT-PCR specificity is still significantly underestimated by these alternative analytic approaches. The LCA model assigned a high probability $(>98 \%)$ of a disease-free status to patients with specimens testing positive with RT-PCR only. However several of these patients had additional evidence supporting a diagnosis of bacterial meningitis (i.e. positive latex agglutination tests and elevated CSF leukocyte counts). This apparent misclassification would result in falsely low RT-PCR specificity and overestimated culture and Gram stain sensitivities. This limitation could possibly be addressed with other CRS or LCA models that incorporate additional tests that are likely less affected by antibiotic administration, such as assays for bacterial antigens (i.e. latex agglutination tests and the rapid immunochromatographic test for S. pneumoniae) [20] or CSF indices (i.e. leukocyte, protein, and glucose counts).

Numerous PCR assays have been evaluated for bacterial meningitis diagnosis using species-specific primers [10-12,16,17,21-24] or broad range bacterial PCR [25,26]. While PCR-based assays have played an increasingly important role in meningitis surveillance in Brazil [12] and elsewhere, including the United Kingdom [27], a routine role in clinical practice has not been established. This is likely due to the resources required and empiric treatment regimens that make confirmation less critical. Current automated RT-PCR systems have advantages over traditional PCR that make it attractive in the clinical setting, including rapid availability of results within hours and a closed system that can reduce contamination risk [28].
Although conventional antimicrobial susceptibility testing requires that the pathogen be cultured, RT-PCR identification of bacterial pathogens might allow some narrowing of antibiotic treatment in clinical situations where cultures are negative. Accuracy of RT-PCR testing could be further improved by using newer RT-PCR primers with improved sensitivity [24] or performing secondary testing with other RT-PCR assays when there is an indeterminate result. However, given the potentially severe consequences of misdiagnosis, the strengths and limitations of any test should be carefully considered before recommending new diagnostic strategies.

CSF Gram staining, long considered a mainstay of bacterial meningitis diagnosis [1], is rapid, inexpensive, and requires relatively little training. However, accurate results are highly dependent on the operator's staining and interpretation skills. Previous studies report Gram stain sensitivities as high as $90 \%$ and specificities of $97 \%$ or more $[8,29]$. In the LCA model in this study, Gram staining had a sensitivity of $98.2 \%$ and specificity of 98.7\%. As previously discussed, the likely misclassification of several patients with an isolated positive RT-PCR result as disease-free suggests that the model overestimated Gram stain and culture sensitivity, and to a lesser extent, specificity. However, as an infectious diseases referral center, Couto Maia Hospital often evaluates several patients with suspected meningitis daily, and the experience of the microbiology staff likely contributed to the excellent performance of Gram staining. Given its wide availability, rapid turnaround, low cost, and high accuracy, the value of Gram staining in the clinical workup of suspected meningitis cannot be understated. 
It should be noted that only $S$. pneumoniae, $N$. meningitidis, and $H$. influenzae were considered in this study. The performance of Gram stain and CSF culture in diagnosing other bacterial meningitis pathogens might differ significantly. Furthermore, because there were few $H$. influenzae cases, due to routine Hib vaccination in infants, the test parameter estimates are likely most valid for $S$. pneumoniae and $N$. meningitidis. Also, local clinical testing guidelines may have resulted in a unique study population in this study, as CSF culture was not routinely recommended for CSF specimens not meeting specific criteria. Interestingly, patients included in our final analysis were more likely to have clinical markers of severe illness consistent with bacterial meningitis when compared to those not included due to absent RT-PCR testing. The reasons for this are unclear; however, according to surveillance staff, the most common reasons for CSF not to be tested with RT-PCR were insufficient volume, loss of the specimen when discarded by hospital staff unfamiliar with the surveillance protocol, or frequent unavailability of specimens during a prolonged aseptic meningitis outbreak in 2007, due to prioritized testing at the state public health laboratory. Since test performance can vary with different patient characteristics, evaluation of these tests in other patient populations might result in different performance parameter estimates, and the clinical characteristics of our study population should be considered when interpreting the findings of this study. However, it is important to note that test sensitivity and specificities are intrinsic parameters that would not vary with changes in disease prevalence alone.

\section{Conclusions}

Newer technologies can often provide increasingly sensitive diagnostic tests; however, there is no straightforward method to evaluate their performance when they are more sensitive than the reference standard. Using multiple analytic approaches, we found that RT-PCR assays on CSF specimens were highly accurate for diagnosis of $S$. pneumoniae, N. meningitidis, and $H$. influenzae meningitis. RT-PCR appears to be useful in situations where antibiotics have been administered prior to lumbar puncture, and this can be particularly helpful for surveillance in settings where this is common. This study also reaffirms the value of CSF Gram staining in the evaluation of acute meningitis, and its routine use should continue to be encouraged.

\section{Competing interests}

The authors declare that they have no competing interests.

\section{Authors' contributions}

HMW, BDP, TAC, SWM, and JNR analyzed the data. MGR, LWM, AIK, and JNR conceived and designed the study. JA, TQO, and MCL conducted the case surveillance and collected clinical data. SMC, BHH, MGC, KS, and JNR conducted the laboratory assays. HMW, SMC, BHH, TAC, AIK, SWM, and JNR drafted the manuscript. All authors read and approved the final manuscript.

\section{Acknowledgements}

We thank the Bahia State Secretary of Health, Salvador, Brazil; and the physicians and staff of Couto Maia Hospital, Salvador, Brazil.

\section{Author details}

${ }^{1}$ Division of Bacterial Diseases, National Center for Immunization and Respiratory Diseases, CDC, 1600 Clifton Road, Atlanta, GA 30333, USA. ${ }^{2}$ Oswaldo Cruz Foundation, Rua Waldemar Falcão 121, Candeal, Salvador Bahia 40296-710, Brazil. ${ }^{3}$ Federal University of Bahia, Salvador, Bahia, Brazil. ${ }^{4}$ Weill Medical College of Cornell University, 1300 York Avenue, New York, NY 10065, USA. ${ }^{5}$ Present address: 550 Peachtree Street NE, MOT 7, Atlanta, GA 30308, USA

Received: 6 September 2012 Accepted: 17 January 2013

Published: 22 January 2013

\section{References}

1. Brouwer MC, Tunkel AR, van de Beek D: Epidemiology, diagnosis, and antimicrobial treatment of acute bacterial meningitis. Clin Microbiol Rev 2010, 23:467-492.

2. Bryan JP, de Silva HR, Tavares A, Rocha H, Scheld WM: Etiology and mortality of bacterial meningitis in northeastern Brazil. Rev Infect Dis 1990, 12:128-135.

3. Bohr V, Rasmussen N, Hansen B, Kjersem H, Jessen O, Johnsen N, Kristensen HS: 875 Cases of bacterial meningitis: diagnostic procedures and the impact of preadmission antibiotic therapy. Part III of a three-part series. J Infect 1983, 7:193-202.

4. Andersen J, Backer V, Voldsgaard P, Skinhoj P, Wandall JH: Acute meningococcal meningitis: analysis of features of the disease according to the age of 255 patients. Copenhagen Meningitis Study Group. J Infect 1997, 34:227-235

5. Dalton HP, Allison MJ: Modification of laboratory results by partial treatment of bacterial meningitis. Am J Clin Pathol 1968, 49:410-413.

6. Nigrovic LE, Malley R, Macias CG, Kanegaye JT, Moro-Sutherland DM, Schremmer RD, Schwab SH, Agrawal D, Mansour KM, Bennett JE, et al: Effect of antibiotic pretreatment on cerebrospinal fluid profiles of children with bacterial meningitis. Pediatrics 2008, 122:726-730.

7. Geiseler PJ, Nelson KE, Levin S, Reddi KT, Moses VK: Community-acquired purulent meningitis: a review of 1,316 cases during the antibiotic era, 1954-1976. Rev Infect Dis 1980, 2:725-745

8. van de Beek D, de Gans J, Spanjaard L, Weisfelt M, Reitsma JB, Vermeulen $\mathrm{M}$ : Clinical features and prognostic factors in adults with bacterial meningitis. N Engl J Med 2004, 351:1849-1859.

9. Karandanis D, Shulman JA: Recent survey of infectious meningitis in adults: review of laboratory findings in bacterial, tuberculous, and aseptic meningitis. South Med J 1976, 69:449-457.

10. Bryant PA, Li HY, Zaia A, Griffith J, Hogg G, Curtis N, Carapetis JR: Prospective study of a real-time PCR that is highly sensitive, specific, and clinically useful for diagnosis of meningococcal disease in children. J Clin Microbiol 2004, 42:2919-2925.

11. Corless CE, Guiver M, Borrow R, Edwards-Jones V, Fox AJ, Kaczmarski EB: Simultaneous detection of Neisseria meningitidis, Haemophilus influenzae, and Streptococcus pneumoniae in suspected cases of meningitis and septicemia using real-time PCR. J Clin Microbiol 2001, 39:1553-1558

12. Sacchi CT, Fukasawa LO, Goncalves MG, Salgado MM, Shutt KA, Carvalhanas TR, Ribeiro AF, Kemp B, Gorla MC, Albernaz RK, et al: Incorporation of real-time $P C R$ into routine public health surveillance of culture negative bacterial meningitis in Sao Paulo Brazil. PLoS One 2011, 6:e20675.

13. Baughman AL, Bisgard KM, Cortese MM, Thompson WW, Sanden GN, Strebel PM: Utility of composite reference standards and latent class analysis in evaluating the clinical accuracy of diagnostic tests for pertussis. Clin Vaccine Immunol 2008, 15:106-114.

14. Hadgu A, Dendukuri N, Hilden J: Evaluation of nucleic acid amplification tests in the absence of a perfect gold-standard test - a review of the statistical and epidemiologic issues. Epidemiology 2005, 16:604-612.

15. Cordeiro SM, Neves AB, Ribeiro CT, Petersen ML, Gouveia EL, Ribeiro GS, Loba TS, Reis JN, Salgado KM, Reis MG, Ko Al: Hospital-based surveillance of meningococcal meningitis in Salvador, Brazil. T Roy Soc Trop Med H 2007, 101:1147-1153. 
16. Carvalho Mda G, Tondella ML, McCaustland K, Weidlich L, McGee L, Mayer LW, Steigerwalt A, Whaley M, Facklam RR, Fields B, et al: Evaluation and improvement of real-time $P C R$ assays targeting lyt $A$, ply, and psaA genes for detection of pneumococcal DNA. J Clin Microbiol 2007, 45:2460-2466

17. Mothershed EA, Sacchi CT, Whitney AM, Barnett GA, Ajello GW, Schmink S, Mayer LW, Phelan M, Taylor TH Jr, Bernhardt SA, et al: Use of real-time PCR to resolve slide agglutination discrepancies in serogroup identification of Neisseria meningitidis. J Clin Microbiol 2004, 42:320-328.

18. Wang X, Hatcher C, Mair R, Theodore J, Kirsch M, Carvalho M, Pimenta F, Dolan J, Harcourt BH, Edmond $\mathrm{K}$, et al: Protein D gene as a target for detection of Haemophilus influenzae. In 109th ASM general meeting. Pennsylvania: Philadelphia; 2009.

19. DeBiasi RL, Tyler KL: Polymerase chain reaction in the diagnosis and management of central nervous system infections. Arch Neurol 1999, $56: 1215-1219$

20. Werno AM, Murdoch DR: Laboratory diagnosis of invasive pneumococcal disease. Clin Infect Dis 2008, 46:926-932.

21. Bronska E, Kalmusova J, Dzupova O, Maresova V, Kriz P, Benes J: Dynamics of PCR-based diagnosis in patients with invasive meningococcal disease. Clin Microbiol Infect 2006, 12:137-141.

22. Richardson DC, Louie L, Louie M, Simor AE: Evaluation of a rapid PCR assay for diagnosis of meningococcal meningitis. J Clin Microbiol 2003, 41:3851-3853.

23. $\mathrm{Ni} \mathrm{H}$, Knight Al, Cartwright K, Palmer WH, McFadden J: Polymerase chain reaction for diagnosis of meningococcal meningitis. Lancet 1992, 340:1432-1434.

24. Wang X, Mair R, Hatcher C, Theodore MJ, Edmond K, Wu HM, Harcourt BH, Carvalho Mda G, Pimenta F, Nymadawa P, et al: Detection of bacterial pathogens in Mongolia meningitis surveillance with a new real-time PCR assay to detect Haemophilus influenzae. Int J Med Microbiol 2011, 301:303-309.

25. Schuurman T, de Boer RF, Kooistra-Smid AM, van Zwet AA: Prospective study of use of PCR amplification and sequencing of $16 \mathrm{~S}$ ribosomal DNA from cerebrospinal fluid for diagnosis of bacterial meningitis in a clinical setting. J Clin Microbiol 2004, 42:734-740.

26. Saravolatz LD, Manzor O, VanderVelde N, Pawlak J, Belian B: Broad-range bacterial polymerase chain reaction for early detection of bacterial meningitis. Clin Infect Dis 2003, 36:40-45.

27. Gray SJ, Trotter CL, Ramsay ME, Guiver M, Fox AJ, Borrow R, Mallard RH, Kaczmarski EB: Epidemiology of meningococcal disease in England and Wales 1993/94 to 2003/04: contribution and experiences of the Meningococcal Reference Unit. J Med Microbiol 2006, 55:887-896.

28. Mothershed EA, Whitney AM: Nucleic acid-based methods for the detection of bacterial pathogens: present and future considerations for the clinical laboratory. Clin Chim Acta 2006, 363:206-220

29. Tunkel AR: Bacterial meningitis. Philadelphia: Lippincott Williams \& Wilkins; 2001.

doi:10.1186/1471-2334-13-26

Cite this article as: Wu et al:: Accuracy of real-time PCR, Gram stain and culture for Streptococcus pneumoniae, Neisseria meningitidis and Haemophilus influenzae meningitis diagnosis. BMC Infectious Diseases 2013 13:26.

\section{Submit your next manuscript to BioMed Central and take full advantage of:}

- Convenient online submission

- Thorough peer review

- No space constraints or color figure charges

- Immediate publication on acceptance

- Inclusion in PubMed, CAS, Scopus and Google Scholar

- Research which is freely available for redistribution 